\title{
Restauratorenausbildung an der Bayerischen Staatsbibliothek
}

\author{
Von der Fachakademie zur Kooperation mit der Technischen \\ Universität München
}

Die Bayerische Staatsbibliothek gehört mit ihrem reichen und einzigartigen schriftlichen Kulturerbe zu den weltweit bedeutenden Universalbibliotheken und Gedächtnisinstitutionen. Daher ist es der Bayerischen Staatsbibliothek eine besondere Sorge und Verpflichtung, die ihr anvertrauten Werke für die wissenschaftliche Erforschung und öffentliche Präsentation in Ausstellungen dauerhaft zu bewahren und zugänglich zu halten. Für diese verantwortungsvolle Aufgabe hat die Bayerische Staatsbibliothek das Institut für Buch- und Handschriftenrestaurierung (IBR) als Fachabteilung für Konservierung/Restaurierung eingerichtet, die auch Dienstleistungen für die regionalen Staatlichen Bibliotheken Bayerns mit wertvollen und einzigartigen historischen Beständen anbietet. Neben der Schadensprävention und der Restaurierung zählen die angewandte Forschung sowie die Ausbildung von Restauratoren seit jeher zum Aufgabenprofil des IBR. Das Institut ging in der Wiederaufbauphase nach dem Zweiten Weltkrieg aus der Technischen Abteilung mit der „Wiederinstandsetzungsstelle fliegergeschädigter Werke der Bayerischen Staatsbibliothek München“ hervor. Als das IBR in den Nachkriegsjahren mit der Entwicklung von Restaurierungsmethoden und mit der Restaurierung bedeutender Werke auch anderer Bibliotheken bekannt geworden war, erhielt die Bayerische Staatsbibliothek zahlreiche Anfragen nach Praktika. Zwischen 1951 und 1971 wurden 76 Buchbinder aus der ganzen Bundesrepublik für mehrwöchige Einführungen in das „Handwerk des Restaurierens“ aufgenommen. Mit dem Bezug einer großen, hochmodernen Werkstatt im Jahr 1963 waren im IBR nunmehr optimale Bedingungen auch für die Fortentwicklung von Restaurierungsmethoden gegeben.

Das weitere Engagement der Bayerischen Staatsbibliothek für die Ausbildung von Restauratoren steht bis heute im engen Kontext mit der Ausdifferenzierung des Restauratorenberufs sowie mit bildungs- und hochschulpolitischen Entwicklungen. Die Bayerische Staatsbibliothek war daher in den vergangenen Jahren mehrfach vor die Herausforderung gestellt, den institutionellen Rahmen und die fachlichen Inhalte der Ausbildung den neuen Anforderungen gemäß fortzuschreiben. 


\section{Gründung der Fachakademie}

Seit den 1960er Jahren wurde in Fachkreisen aller Restaurierungssparten die Notwendigkeit einer geregelten Ausbildung zum Restaurator formuliert. Für die Fachrichtungen Gemälde und Skulptur wurden in den 1970er Jahren an den Kunstakademien in Dresden und Stuttgart Diplomstudiengänge für Restaurierung geschaffen. Papierund Graphikrestaurierung konnte ab 1966 an der Akademie der bildenden Künste in Wien in enger Anbindung an die Österreichische Nationalbibliothek studiert werden, einer $\mathrm{zu}$ jener Zeit weltweit einzigartigen und höchst attraktiven Ausbildungsmöglichkeit, die viele Hospitanten und Praktikanten anzog. Über Form und Inhalt der Ausbildung für den sich aus dem Buchbinderhandwerk fortentwickelnden Beruf des Buch- und Papierrestaurators wurde vielfach auch in Deutschland diskutiert. Um das Jahr 1990 entstanden fast gleichzeitig drei Ausbildungseinrichtungen für diesen Bereich: an der Staatlichen Akademie der Bildenden Künste Stuttgart, der Fachhochschule Köln und der Bayerischen Staatsbibliothek München. Zehn Jahre später kam noch die Fachhochschule Hildesheim hinzu. Die Einrichtung von Studiengängen an Hoch- und Fachhochschulen entsprach der berufsständischen Forderung nach einer akademischen Restauratorenausbildung. Mit Verordnung des Bayerischen Staatsministeriums für Unterricht und Kultus vom 19. Januar 1990 wurde die Staatliche Fachakademie für die Ausbildung von Restauratoren für Archiv- und Bibliotheksgut an der Bayerischen Staatsbibliothek in der Trägerschaft des Freistaats Bayern gegründet. Kerngedanke dieses bayerischen Ausbildungsmodells war die enge Verzahnung von praktischer und theoretischer Ausbildung, die auf eine vorhergehende Berufsausbildung aufbaut und auf eine gehobene Berufslaufbahn vorbereitet. Mit der Staatlich anerkannten Fachakademie zur Ausbildung von Restauratoren für Möbel und Holzobjekte des A. R. Goering Instituts e. V. bestand seit 1987 eine weitere einschlägige Fachakademie in München.

Die Gründung der Fachakademie markierte den Beginn einer staatlich geregelten und kontinuierlichen Restauratorenausbildung an der Bayerischen Staatsbibliothek. Die Initiative geht auf den damaligen Leiter des Instituts für Buch- und Handschriftenrestaurierung Dr. Helmut Bansa zurück, der in seinen Bemühungen von der damaligen Generaldirektion der Bayerischen Staatlichen Bibliotheken unter Dr. Eberhard Dünninger und vom damaligen Direktor der Bayerischen Staatsbibliothek Dr. Franz Georg Kaltwasser unterstützt wurde. Für Unterricht, Organisation und Verwaltung wurden der Fachakademie innerhalb des Haushalts des Ministeriums für Unterricht und Kultus entsprechende Personal- und Sachmittel zugewiesen. Der Institutsleiter wurde für die Leitung der Fachakademie teilabgeordnet, der dadurch in die ungewöhnliche Lage kam, zwei Dienstherren unterstellt zu sein: dem Staatsminister für Wissenschaft, Forschung und Kunst sowie dem Staatsminister für Unterricht und Kultus.

Die Fachakademie bot als eine berufsbildende Einrichtung im bayerischen Ausbildungssystem in einer dreijährigen Vollzeitausbildung die Weiterqualifizierung 
des gelernten Handbuchbinders zum „Staatlich geprüften Restaurator“. Aufnahmebedingungen für die Fachakademie waren demnach ein mittlerer Schulabschluss, eine einschlägige abgeschlossene Berufsausbildung sowie das erfolgreiche Bestehen eines Aufnahmeverfahrens und einer sechsmonatigen Probezeit. Für jeden Studiengang wurden nach diesen Vorgaben aus den jeweils gut zwanzig Bewerbern die sechs besten ausgewählt. In der Aufnahmeprüfung mussten die Kandidaten die damalige Chefrestauratorin des Instituts, Frau Luise Karl, davon überzeugen, dass ihre praktischen Fähigkeiten mindestens dem Ausbildungsstand entsprechend entwickelt und noch wesentlich weiter ausbaufähig waren. Die ausgewählten Kandidaten verfügten nach einer dreijährigen Ausbildung zum Handbuchbinder allesamt über den Gesellenbrief, einige davon als Bundes- oder Landessieger, zwei hatten zusätzlich den Meisterbrief. Dreiviertel aller Studierenden brachten die Hochschulreife, weitere zwanzig Prozent die Fachhochschulreife mit. Da sich die Restaurierung über die Zeit zum Frauenberuf entwickelt hat, machten Bewerberinnen den Löwenanteil aus. Unter den insgesamt 36 Studierenden der sechs Studiengänge der Fachakademie zwischen 1991 und 2009 waren jedoch immerhin sechs angehende Restauratoren.

Der erste Studiengang begann im September 1991 und schloss im Juli 1994 mit Unterrichts- und Ferienzeiten gemäß dem bayerischen Schuljahr. Der Anzahl an Ausbildungsplätzen entsprechend begann erst wieder ein neuer Studiengang, wenn die jeweils sechs Studierenden nach drei Ausbildungsjahren als Staatlich geprüfte Restauratoren in das Berufsleben entlassen waren. Die Studierenden wurden an fünf Tagen der Woche im IBR ausgebildet, in dem sie sich nach einer kurzen Eingewöhnungsphase voll integriert fühlten. Auch umgekehrt - aus der Sicht des IBR - gehörten die Schüler „dazu“, obwohl Engpässe in der Raumbelegung trotz guter Planung unvermeidbar waren. Der theoretische Unterricht fand daher in einer auf die kleine Gruppe wie zugeschnittenen Mansarde in der Kaulbachstraße 19 statt, einem Nebengebäude der Bayerischen Staatsbibliothek. Das Ausbildungsprogramm umfasste in den drei Studienjahren insgesamt 4.560 Unterrichtsstunden, die zu etwa gleichen Teilen aus Unterricht in praktischer Restaurierung und in naturwissenschaftlichen oder kulturgeschichtlichen Fächern bestanden. Die Studierenden lernten von Beginn ihrer Ausbildung an das Restaurieren an Originalen der Bayerischen Staatsbibliothek. Der Unterrichtsplan sah die Restaurierung von jeweils ein bis zwei Exemplaren der Hauptgattungen von Bucheinbänden und Papieren vor. Gruppenprojekte galten etwa der Konservierung von historischen Fotografien und Papyri oder auch Themen aus der angewandten Forschung zu restaurierungspraktischen Methoden. Die Kooperation mit dem direkt benachbarten Bayerischen Hauptstaatsarchiv erweiterte den Lehrplan des zweiten Studienjahrs um archivspezifische Themen, wie etwa die Restaurierung von Wachssiegeln.

Die praktische und schriftliche Abschlussprüfung dauerte jeweils von März bis Juli, mit drei Sitzungen des Prüfungsausschusses unter dem Vorsitz des Kultusministeriums und der Regierung von Oberbayern als vorgeordnete Schulaufsichtsbehörden. Die Abschlussfeierlichkeiten standen stets unter der Schirmherrschaft einer promi- 
nenten Persönlichkeit wie Seiner Königlichen Hoheit Herzog Franz von Bayern oder des damaligen Bayerischen Staatsministers der Finanzen Prof. Dr. Kurt Faltlhauser. Der feierliche Festakt im Fürsten- oder Marmorsaal der Bayerischen Staatsbibliothek begann mit der Begrüßung der etwa 150 geladenen Gäste durch den Generaldirektor, der sich Grußworte von Vertretern des Kultusministeriums sowie Reden der damaligen Chefrestauratorin des Instituts Luise Karl und der Leitung der Fachakademie verbunden mit der Vergabe der Zeugnisse anschlossen. Die Instituts- und Fachakademieleitung lagen bis 2001 bei Dr. Helmut Bansa, danach bei Dr. Irmhild Schäfer als seiner Nachfolgerin in den beiden miteinander verbundenen Funktionen. Ein weiterer Höhepunkt der Feier war stets die Verleihung des Meisterpreises der Bayerischen Staatsregierung an die jeweils besten Absolventinnen und Absolventen durch Christl Beinhofer als stellvertretender Leiterin. Feste Bestandteile der Abschlussfeier waren auch eine Ausstellung von restaurierten Objekten und eine Publikation über Restaurierungsprojekte aus den drei Studienjahren.

\section{Fachakademie und Bologna-Prozess}

Die seit Ende der 1990er Jahre europaweit einsetzende hochschulpolitische Entwicklung in Deutschland und ganz Europa hat zu einer Umstrukturierung der Restauratorenausbildung an den Hochschulen und Fachhochschulen wie auch an der Bayerischen Staatsbibliothek geführt. Grundlage ist die von 29 europäischen Bildungsministern im Jahr 1999 im italienischen Bologna abgegebene politisch-programmatische Erklärung zur Schaffung eines einheitlichen Europäischen Hochschulraums bis zum Jahr 2010. Ziele dieser Hochschulreform sind die europaweite Harmonisierung von Studiengängen und -abschlüssen sowie die internationale Mobilität der Studierenden und Lehrenden. Mit der Novellierung des Hochschulrahmengesetzes seit 1998 wurden die deutschen Hochschulen dazu verpflichtet, bis spätestens 2010 die international anerkannten Hochschulgrade Bachelor und Master einzuführen. Bisher verliehen die deutschsprachigen Ausbildungsstätten nach einer Studiendauer von vier bis fünf Jahren das Diplom. Im Zuge des sog. Bologna-Prozesses wurde für fast alle Restaurierungsstudiengänge an Hoch- und Fachhochschulen der Bachelor of Arts (B. A.) nach drei oder vier Jahren als erster berufsqualifizierender Abschluss eingeführt, auf den der zweijährige Master of Arts (M. A.) folgt.

Damit den Absolventinnen und Absolventen der Fachakademie-Ausbildung keine beruflichen Nachteile entstehen, sollten auf der Grundlage eines Beschlusses des Bayerischen Landtags verschiedene Optionen ihrer Eingliederung in eine Fachhochschule geprüft werden. Gemeinsam mit Ministeriumsvertretern wurden daher ab Herbst 2005 Sondierungsgespräche zunächst mit der Fachhochschule Weihenstephan und der Fachhochschule München mit ihrer Studienrichtung Papiertechnik 
geführt, die jedoch aufgrund unterschiedlicher konzeptioneller Vorstellungen nicht zum Ergebnis führten.

Der Bologna-Prozess bot aber noch eine weitere Option für die geplante Umstrukturierung der Fachakademie-Ausbildung. Die Umstellung der Diplomstudiengänge auf Bachelor- und Masterstudiengänge an der Technischen Universität München (TUM) spätestens zum Wintersemester 2009/2010 betraf auch den dortigen Diplomstudiengang für „Restaurierung, Kunsttechnologie und Konservierungswissenschaft“. Die Technische Universität München und die Bayerische Staatsbibliothek sahen in den zukünftigen Studienstrukturen eine erfolgversprechende Möglichkeit zur Eingliederung der Fachakademie in die neu zu konzipierenden Studiengänge. Der Lehrstuhlinhaber, Professor Dipl.-Rest. Erwin Emmerling, brachte sein Interesse an einer Kooperation mit der Bayerischen Staatsbibliothek mit dem Ziel einer inhaltlich weiter gefassten Ausgestaltung der geplanten Studiengänge deutlich zum Ausdruck. Auf der Grundlage dieser Entwicklungen entschied das zuständige Ministerium, an der Staatlichen Fachakademie für Archiv- und Bibliotheksgut ab Herbst 2006 letztmalig einen Studiengang beginnen zu lassen. Parallel dazu erfolgten ab 2008 mit Hochdruck die gemeinsamen Planungen von Fachakademie und Lehrstuhl für die Neukonzeption der Ausbildung im Bereich Grafik, Archiv- und Bibliotheksgut als Bestandteil des neuen Bachelorstudiengangs ab dem Wintersemester 2009/2010.

Mit der Abschlussfeier im Juli 2009 wurde der letzte Studiengang der Fachakademie in das Berufsleben entlassen. Die Staatlich geprüften Restauratorinnen und Restauratoren haben wie ihre Vorgänger zeitnah zur Abschlussprüfung attraktive Anstellungen bekommen: am Theatermuseum in München, an der Staatsgalerie Stuttgart, der Universitätsbibliothek der Technischen Universität München, am Literaturarchiv Marbach am Neckar und an der Bayerischen Staatsbibliothek. Von 1991 bis 2009 wurden in sechs Studiengängen insgesamt 36 Restauratorinnen und Restauratoren ausgebildet, die in verschiedenen Bundesländern an staatlichen Bibliotheken, Archiven, Museen oder freiberuflich mit eigenen Werkstätten tätig sind. In seinem Grußwort auf der Abschlussfeier des Studiengangs 2003/2006 sprach Dr. Rolf Griebel in seiner Doppelrolle als Generaldirektor der Bayerischen Staatsbibliothek und „Hausherr“ der Fachakademie von der Verpflichtung der Bayerischen Staatsbibliothek mit ihren reichen und einzigartigen Beständen, restauratorischen Nachwuchs heranzubilden. Aus jedem der sechs Studiengänge konnten Absolventen an der Bayerischen Staatsbibliothek angestellt werden, so dass heute mit acht der insgesamt 36 zwischen 1991 und 2009 ausgebildeten Restauratoren fast 25 \% der Absolventen am IBR tätig sind.

Die Abschlussfeier im Jahr 2009 markierte zugleich den Abschied von achtzehn Jahren Fachakademie wie den Neubeginn der Restauratorenausbildung in einem veränderten institutionellen Rahmen und mit neuen Inhalten. 


\section{Institutionelle Kooperation mit der Technischen Universität München}

Auf der Grundlage der vorausgegangenen Entwicklung entschieden die Bayerischen Staatsminister für Unterricht und Kultus und für Wissenschaft, Forschung und Kunst im Januar 2009, die Ausbildung an der Staatlichen Fachakademie für Restaurierung von Archiv- und Bibliotheksgut nicht mehr fortzuführen. An ihre Stelle trat eine grundlegend neu konzipierte Ausbildung in Form eines inhaltlichen Studienschwerpunkts „Buch und Papier“ im neuen Bachelor- und zukünftigen Masterstudiengang „Restaurierung, Kunsttechnologie und Konservierungswissenschaft“ an der Technischen Universität München (TUM) zum Wintersemester 2009/2010. Als Grundlage für diese enge institutionelle Zusammenarbeit wurde zwischen der Bayerischen Staatsbibliothek und der TUM eine formelle schriftliche Vereinbarung getroffen. Die vom Präsidenten der Technischen Universität Professor Dr. Wolfgang Herrmann und vom Generaldirektor der Bayerischen Staatsbibliothek Dr. Rolf Griebel unterzeichnete Kooperationsvereinbarung trat am 1. Oktober 2009 in Kraft. Gegenstand der Zusammenarbeit ist „die wissenschaftliche Zusammenarbeit in Forschung und Lehre und die praktische Konservierung und Restaurierung im Rahmen des Studienganges ,Restaurierung, Kunsttechnologie und Konservierungswissenschaft‘. Die Unterzeichner verstehen diese Vereinbarung als Chance und Aufgabe, eine herausragende praxisbezogene Zusammenarbeit in der modernen Konservierungswissenschaft zu begründen und in beispielhafter Kooperation optimal zu fördern und zu unterstützen“ (§ 1, Abs. 1 und 2). Die Zuständigkeit zur Durchführung dieser Zusammenarbeit liegt auf Seiten der Bayerischen Staatsbibliothek bei der Leitung des Instituts für Buch- und Handschriftenrestaurierung, auf Seiten der TUM beim Inhaber des Lehrstuhls für „Restaurierung, Kunsttechnologie und Konservierungswissenschaft“. Die Personal- und Sachmittel der Fachakademie wurden mit Zweckbindung in den Haushalt der Bayerischen Staatsbibliothek übertragen.

Der Lehrstuhl für „Restaurierung, Kunsttechnologie und Konservierungswissenschaft“ ist Teil des Instituts für Baugeschichte, Kunstgeschichte und Restaurierung an der Fakultät für Architektur. Mit den drei weiteren Lehrstühlen des Instituts bestehen enge fachliche und personelle Verbindungen in Forschung und Lehre, etwa mit Vorlesungen für Restauratoren zur Kunstgeschichte und Denkmalpflege. Dazu zählen der Lehrstuhl für Baugeschichte, Historische Bauforschung und Denkmalpflege, der Lehrstuhl für Architekturgeschichte und kuratorische Praxis sowie der Lehrstuhl für Theorie und Geschichte von Kunst und Design. Der Lehrstuhlinhaber für Architekturgeschichte ist in Personalunion Direktor des Architekturmuseums der TUM mit der deutschlandweit größten und bis in das 16. Jahrhundert zurückreichenden Sammlung von Architekturplänen und Nachlässen bedeutender Architekten. 


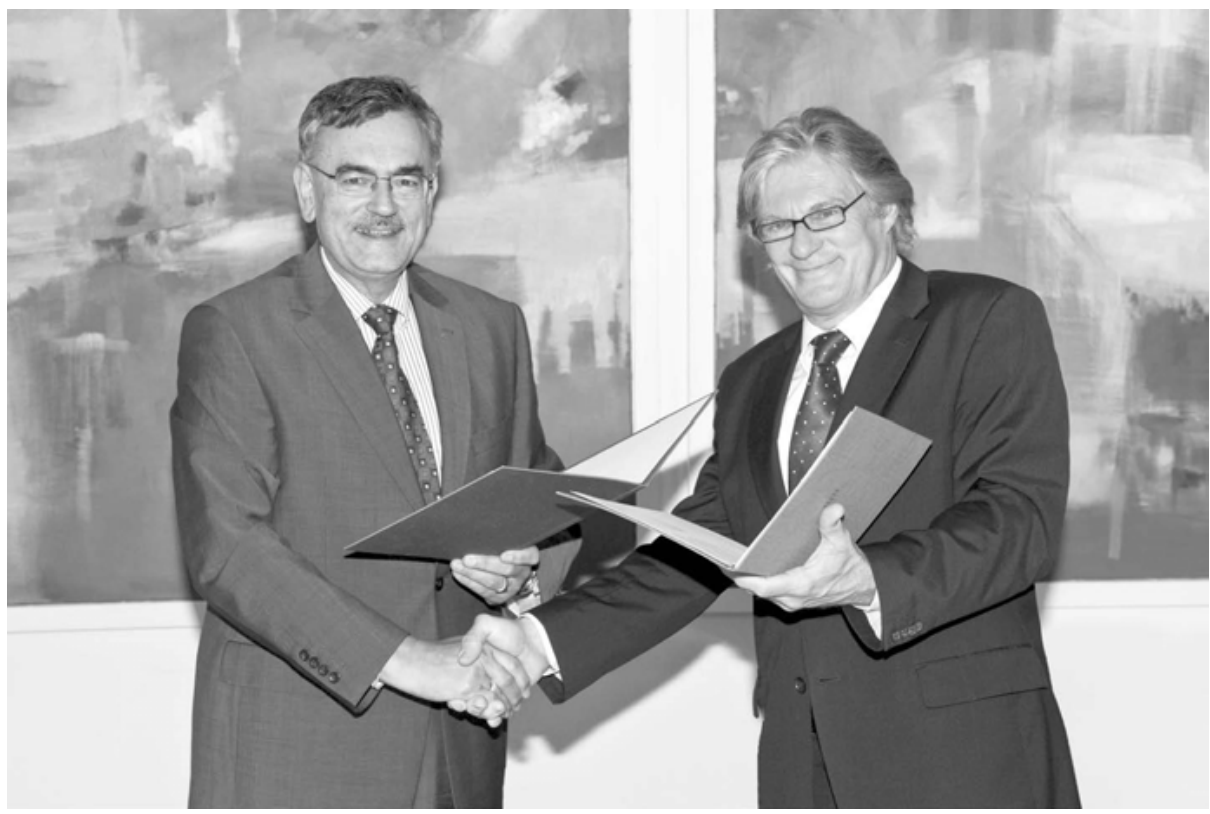

Abb. 1: Prof. Dr. Herrmann und Dr. Rolf Griebel mit der unterzeichneten Kooperationsvereinbarung

Eine Besonderheit des Lehrstuhls für „Restaurierung, Kunsttechnologie und Konservierungswissenschaft“ besteht in den vielfältigen Kooperationen, auch über die benachbarten Lehrstühle und die Fakultäten für Physik und Chemie hinaus. Seit seiner Gründung im Jahr 1996 arbeitet der Lehrstuhl in der Restauratorenausbildung mit der Archäologischen Staatssammlung, dem Bayerischen Nationalmuseum, dem Bayerischen Landesamt für Denkmalpflege und den Bayerischen Staatsgemäldesammlungen mit ihrem Doerner-Institut zusammen. Enge Kooperationen bestehen ferner mit der Bayerischen Verwaltung der staatlichen Schlösser, Gärten und Seen, dem Deutschen Museum, dem Völkerkundemuseum und vielen anderen staatlichen Sammlungen und Museen in München, ganz Bayern und anderen Bundesländern. Projekte des Lehrstuhls gelten der Denkmalpflege in Bayern wie etwa dem ehemaligen Zisterzienserkloster Raitenhaslach bei Burghausen oder Untersuchungen und Dokumentationen in Schloss Schwarzenberg bei Scheinfeld (Landkreis Neustadt an der Aisch-Bad Windsheim). In internationalen Projekten kooperiert der Lehrstuhl mit Institutionen in China (Terrakotta-Armee) und Afghanistan (von den Taliban zerstörte Buddha-Statuen). Kürzlich wurde ein neues Projekt unter Federführung der Technischen Universität und der Fraunhofer-Gesellschaft zusammen mit weiteren europäischen Forschungsinstitutionen ins Leben gerufen, das sich der beispielhaften Restaurierung und Konservierung eines Gebäudekomplexes mit Wandbildern in der antiken, durch den Vesuvausbruch im Jahr 79 n. Chr. untergegangenen Stadt Pompeji widmet. 
Dank der Kooperationen mit den Münchener Institutionen können die Studierenden in deren Restaurierungsabteilungen in Theorie und Praxis ausgebildet werden und interdisziplinär in allen Fachrichtungen studieren. Lehre wie praktische Ausbildung erfolgen durch die fachspezifischen Beiträge dieser Einrichtungen mit ihren Restaurierungswerkstätten. Dadurch erhalten die künftigen Restauratoren für Grafik, Bibliotheks- und Archivgut neben einer umfassenden naturwissenschaftlichen Ausbildung Einblicke in angrenzende Bereiche, wie etwa die Konservierungswissenschaft im Bereich Holz und Textil. Der Lehrstuhl mit Vortragsräumen, Werkstätten und Labor hat seinen Sitz in dem im Jahr 2000 eingerichteten Werkstättengebäude von Bayerischem Nationalmuseum und Archäologischer Staatssammlung in der Oettingenstraße im alten Stadtteil Lehel. Für die Vorlesungen ist der Lehrstuhl von der Bayerischen Staatsbibliothek aus in einer Viertelstunde zu Fuß quer durch den Englischen Garten gut erreichbar. Eine gleichsam universitäre Campus-Situation bietet sich den Studierenden mit Blick auch auf die anderen nahegelegenen Kooperationspartner wie Bayerisches Nationalmuseum, Bayerisches Landesamt für Denkmalpflege und Bayerische Staatsgemäldesammlungen mit dem Doerner-Institut. In unmittelbarer Nachbarschaft liegen auch die Staatliche Graphische Sammlung, das Architekturmuseum und das Münchener Stadtmuseum mit ihren Restaurierungsabteilungen, so dass man insgesamt geradezu von einem „Restaurierungscampus“ sprechen kann.

\section{„Buch und Papier“ im Bachelorstudiengang}

Der neue Bachelorstudiengang „Restaurierung, Kunsttechnologie und Konservierungswissenschaft“ begann plangemäß im Wintersemester 2009/2010 und damit zeitlich parallel zum auslaufenden zehnsemestrigen Diplomstudiengang. Zwanzig Studierende nahmen in diesem Studiengang ihr Studium an der TUM auf. Der Bachelorstudiengang beginnt stets zum Wintersemester und hat eine Regelstudienzeit von insgesamt acht Semestern inklusive zwei Auslandssemestern. Neben den allgemeinen Zugangsvoraussetzungen für ein Studium ist der Nachweis eines mindestens zwölfmonatigen Praktikums in einer Restaurierungswerkstätte, zum Beispiel eines Museums, einer Bibliothek, eines Archivs oder Denkmalamts des In- und Auslandes, notwendig. Das sog. Vorpraktikum kann alternativ auch in einer privaten Restaurierungswerkstätte absolviert werden. Zusätzlich ist der Nachweis der Eignung im Rahmen einer Eignungsfeststellung mit einer eingereichten Dokumentation zum Vorpraktikum und im Auswahlgespräch zu erbringen. Das Studium ist modular mit Veranstaltungen im Pflicht-, Wahlpflicht- und Wahlbereich aufgebaut. Für jedes Modul erhält der Studierende eine festgelegte Zahl an Anrechnungspunkten oder Credits (nach dem European Credit Transfer System ECTS), wenn es erfolgreich bestanden ist. Credits sind gemäß der Kultusministerkonferenz (KMK) ein quantitatives Maß für die arbeitsmäßige Gesamtbelastung von Studierenden, bei deren Berechnung der 
Besuch von Lehrveranstaltungen (Präsenzzeit), die Vor- und Nachbereitungszeiten, die Prüfungsvorbereitung sowie das Anfertigen von Referaten, Haus- und Projektarbeiten berücksichtigt werden. Auf der Grundlage des Beschlusses der KMK vom 24.10.1997 wird für einen Credit eine Arbeitsbelastung (work load) des Studierenden im Präsenz- und Selbststudium von 30 Stunden angenommen. Somit sind pro Semester 30 Credits zu veranschlagen (40 Stunden/Woche, 22,5 Wochen).

Insgesamt sind in den acht Semestern mindestens 240 Credits $\mathrm{zu}$ erbringen, bevor nach einer bestandenen Bachelorprüfung mit Vortrag über die Thesis der akademische Grad „Bachelor of Arts“ (B. A.) verliehen werden kann. Durch Lehrveranstaltungen können mindestens 180 Credits erworben werden. Da die Studierenden an internationale Spitzenstandards herangeführt werden sollen, gehört ein zweisemestriger Auslandsaufenthalt im Umfang von bis zu 45 Credits zum Pflichtumfang dieses Studiums. Für die dreimonatige Bachelor's Thesis mit Kolloquium im achten Semester gibt es 15 Credits. Der akademische Grad soll mit dem Hochschulzusatz (TUM) geführt werden.

Studierende, die sich für den Studienschwerpunkt „Buch und Papier“ entscheiden, belegen die thematisch übergreifenden, für alle Restaurierungssparten grundlegenden Lehrveranstaltungen des Lehrstuhls und seiner Kooperationspartner sowie ergänzend die fachspezifischen Veranstaltungen von Dozenten des IBR. Die praktischen Übungen für die Schwerpunktstudierenden finden in den Werkstätten des IBR statt, die Vorlesungen und Prüfungen am Lehrstuhl in der Oettingenstraße. An den vom IBR durchgeführten Vorlesungen nehmen auch nahezu alle anderen Studierenden des Lehrstuhls zum Erwerb der entsprechenden Credits teil. Diese duale Ausrichtung des Studiums ermöglicht es den Studierenden, über den Bereich von „Buch und Papier“ hinaus ein breites Basiswissen zu erwerben. Im Weiteren führt dieses Konzept zu den erwarteten Synergieeffekten der Kooperation mit der TUM. Zum Beispiel profitieren „unsere“ Studierenden von Vorlesungen des Doerner-Instituts zur Präventiven Konservierung und von der grundlegenden Ausbildung in Kunsttechnologie und Dokumentation am Lehrstuhl. Insbesondere bei diesen Fächern bestehen große Schnittmengen zwischen den Restaurierungssparten.

Neben den theoretischen Fächern, wie zum Beispiel Kodikologie, Werkstoffkunde und Präventive Konservierung für Bibliotheksgut werden im Bachelorstudiengang die grundlegenden Kenntnisse der Papier- und Einbandrestaurierung vermittelt und weitgehend auch an Praxisbeispielen umgesetzt. Dank der reichen Bestände der Bayerischen Staatsbibliothek können die Studierenden selbst in Spezialgebieten wie islamischer Buchkunst an Originalen lernen. So können direkt im Seminarraum 4000 Jahre alte Keilschriften auf Stein oder ein spätgotischer Originaleinband aus der Gutenberg-Zeit auf seine Fertigungsweise untersucht werden. Spezialthemen zur Restaurierungspraxis werden in mehrtägigen Blockveranstaltungen außerhalb der Vorlesungszeit von externen Dozenten behandelt. Zum Beispiel hält eine Fotorestauratorin ergänzend zur Vorlesung des IBR im Modul Moderne Medien einen Workshop zur Fotokonservierung. Sie vermittelt an Originalen das Erkennen historischer Techniken, 
verbunden mit den jeweils hierzu passenden Maßnahmen, wie zum Beispiel das Reinigen verschiedener fotografischer Oberflächen und das Behandeln von Schadstellen an fotografischen Materialien. Für den von der Ernst von Siemens Kunststiftung mit Stipendien finanziell unterstützten, bisher zweimal durchgeführten Workshop in japanischer Kaschiertechnik konnte ein international renommierter Hyogu-Meister und Restaurator aus Japan gewonnen werden, der die zehnjährige Ausbildung zum Hyogu-Meister an der Oka Bokkodo in Kyoto absolviert hat. Die Oka Bokkodo gehört zum exklusiven Kreis der Werkstätten mit nationaler Lizenz für die Restaurierung des wertvollsten nationalen Kulturerbes. Der Kurs ermöglicht es insgesamt zehn Studierenden, auch von den anderen deutschen Ausbildungsinstitutionen in Stuttgart, Köln und Hildesheim, Grundkenntnisse über traditionelle Kaschiertechniken zu erlernen, und bietet ihnen die Gelegenheit zu Gesprächen mit erfahrenen Restauratoren über die Anwendung japanischer Techniken und Materialien.

Im Sommersemester 2013 fanden nach acht Semestern die Bachelorprüfungen für sechzehn Studierende aus dem ersten Bachelorjahrgang statt, darunter zwei mit dem Studienschwerpunkt „Buch und Papier“. Im Sommersemester 2014 werden die nächsten Bachelorprüfungen bei den knapp zwanzig Studierenden des zweiten Bachelorjahrgangs ab Wintersemester 2010/2011 abgenommen, darunter wieder zwei Schwerpunktstudierende. In der Bachelor's Thesis erarbeiten die Studierenden auf der Grundlage von materialwissenschaftlichen sowie kunsttechnologischen Untersuchungen die Restaurierungs- und Konservierungskonzepte für „ihr“ Objekt und setzen diese um. Zusätzlich zu den Studierenden von „Buch und Papier“ schreiben auch Studierende mit anderen Schwerpunktsetzungen ihre Bachelorarbeit am IBR zu passenden Themen der Kunsttechnologie und Konservierungswissenschaft, zum Beispiel im Sommersemester 2014 ein Student mit Schwerpunkt in der Gemälderestaurierung über ein rein materialwissenschaftliches und kunsttechnologisches Thema bei ausgewählten tibetischen Buchdeckeln. Die Ergebnisse dieser Bachelorarbeit werden in ein laufendes, von der Deutschen Forschungsgemeinschaft gefördertes Projekt der Bayerischen Staatsbibliothek zur Erschließung und Digitalisierung von Buchdeckeln als selbstständige Kunstobjekte einfließen. Es zeigt sich, dass von den knapp zwanzig Studierenden, die jährlich zum Wintersemester ihr Restaurierungsstudium an der TUM aufnehmen, jeweils zwei Studierende ihren Abschluss in unserem Schwerpunkt machen. Während die Vorlesungen des Schwerpunktes von nahezu allen Studierenden besucht werden, sind die Studienplätze in den Werkstätten des IBR begrenzt. Damit bildet das IBR durch den jährlichen Studienbeginn in der Summe genauso viele Restauratoren aus wie zu Zeiten der Fachakademie. 


\section{„Buch und Papier“ im Masterstudiengang}

Zum Wintersemester 2013/2014 begann für die Bachelorabsolventen nahtlos der konsekutive Masterstudiengang „Restaurierung, Kunsttechnologie und Konservierungswissenschaft". Auch Bachelorabsolventen anderer Hochschulen in Deutschland oder im Ausland können sich für den Masterstudiengang bewerben. „Konsekutiv“ bedeutet einschränkend nur, dass die Qualifikation für den Studiengang durch einen Bachelorabschluss in einem mindestens sechssemestrigen Studiengang „Restaurierung/Konservierung“ nachzuweisen ist. Voraussetzung für die Spezialisierung im Studienschwerpunkt „Buch und Papier“ ist ein Bachelorabschluss im Bereich Grafik, Archiv- und Bibliotheksgut. Der Masterstudiengang dient der Vermittlung von vertieften Kenntnissen und Fähigkeiten in zahlreichen Gebieten der Konservierung und Restaurierung. Die Studierenden haben die Möglichkeit, sich entsprechend ihrer Neigung auf ein Fachgebiet zu spezialisieren, wie zum Beispiel einen Studienschwerpunkt auf Buch- und Handschriftenrestaurierung zu legen, auf die Restaurierung von Textilien oder von gefassten Bildwerken. Die Spezialisierung erfolgt durch die Auswahl von Vorlesungen, praktischen Übungen und Projektarbeit. Eine Besonderheit im Masterstudiengang ist, dass jeder Studierende durch einen Mentor in der individuellen Zusammenstellung seines Studienplans begleitet wird. Die Regelstudienzeit beträgt insgesamt vier Semester. Im vierten Semester wird die Master's Thesis erstellt. Für die Erlangung des Mastergrades müssen Lehrveranstaltungen im Gesamtumfang von insgesamt 120 Credits belegt werden. Davon entfallen 30 Credits auf die sechsmonatige Master's Thesis.

Studierende des Studienschwerpunkts „Buch und Papier“ erwerben mit den Credits der spezifischen Lehrveranstaltungen und der Master's Thesis am IBR insgesamt 82 Credits und damit etwa zwei Drittel der Gesamtpunktezahl im Masterstudium. Die übrigen 38 Credits erhalten sie bei bestandenen Prüfungen der thematisch übergreifenden Veranstaltungen des Lehrstuhls und der anderen Kooperationspartner. Wie bereits im Bachelorstudium finden die Vorlesungen des IBR für alle Studierenden eines Jahrgangs des gesamten Masterstudienganges statt. Inhaltlich ergänzen sich die Veranstaltungen des IBR und des Lehrstuhls gegenseitig zu einem in sich konsistenten Konzept der gesamten restauratorischen Ausbildung. Die Module Kunsttechnologie und Kunstgeschichte, Konservierungs-/Restaurierungsmethoden sowie Bestandserhaltung decken zusammen mit den Nebenfächern das Studienangebot für alle beruflichen Anforderungen der Arbeitswelt ab. Darüber hinaus lernen die Studierenden die alltäglichen Herausforderungen im Bereich der Restaurierung, Konservierung und Bestandserhaltung an einer großen Bibliothek unmittelbar am IBR kennen.

Der Masterstudiengang bietet ein wissenschaftlich fundiertes und praxisorientiertes Lehrangebot. Kernfächer des Studiums sind Konservierungswissenschaft und Kunsttechnologie, begleitet von naturwissenschaftlichen und kunstgeschichtlichen Grundlagen. Der zweite Schwerpunkt des Studiums besteht in der Praxis der Restaurierung und Bestandserhaltung. Eigene Restaurierungsprojekte sowie die intensive 
Betreuung in kleinen Gruppen und die Förderung der interdisziplinären Zusammenarbeit am „Restaurierungscampus“ München bieten unseren Studierenden optimale Voraussetzungen für ihre Tätigkeit an Bibliotheken, Archiven, Museen oder als freiberufliche Restauratoren. Besonders geeigneten Studierenden steht mit dem „Master of Arts“ (M. A.) auch der Weg zur Promotion offen. Alle Bachelorabsolventen unseres Schwerpunkts haben zum Wintersemester 2013/2014 das Masterstudium aufgenommen und werden voraussichtlich innerhalb von zwei Jahren ihren Abschluss als Restaurator M. A. (TUM) erreichen. Auch in den folgenden Jahren werden die laufenden Bachelorjahrgänge das Masterstudium aufnehmen.

Zum Wintersemester 2013/2014 erfolgte eine weitere Fokussierung der Restauratorenausbildung in der Zusammenarbeit mit der TUM, die der primären Orientierung des IBR an der hochspezialisierten Bearbeitung und Erforschung von Spitzenbeständen des schriftlichen Kulturerbes in besonderem Maße entgegenkommt. Entsprechend der dezidierten Forschungsorientierung des Lehrstuhls wurde nach längerer Vorbereitungsphase mit diesem Semester der Bachelorstudiengang eingestellt, um den Fokus der Ausbildung ganz auf den Masterstudiengang legen zu können. Neuzulassungen zum Bachelorabschluss sind nun nicht mehr möglich. Die Zulassung zum Studiengang „Restaurierung, Kunsttechnologie und Konservierungswissenschaft“ an der TUM setzt nun vielmehr den Abschluss eines an einer anderen Hochschule absolvierten Bachelorstudiums voraus, das Masterstudium an der TUM umfasst ausschließlich die auf vier Semester angelegte weitere Qualifizierung zum Master und eine gegebenenfalls anschließende Promotion.

Die Restauratorenausbildung im Studienschwerpunkt „Buch und Papier“ an der Bayerischen Staatsbibliothek beginnt also ab dem Wintersemester 2013/2014 mit den Bachelorabsolventen auf einem fortgeschrittenen akademischen Niveau. Diese Studierenden müssen nicht erst allmählich an das praktische Arbeiten mit Objekten herangeführt werden. Damit eröffnen sich ganz neue Möglichkeiten für Restaurierungs- und Forschungsprojekte mit den Studierenden; beispielsweise können neue Verfahren der Restaurierung/Konservierung entwickelt und erforscht sowie in Pilotprojekten praktisch umgesetzt werden. Darüber hinaus können Fragestellungen zu kunsttechnologischen Themenkomplexen, die von Wissenschaftlern an die Bayerische Staatsbibliothek herangetragen werden, vom IBR zusammen mit den Studierenden und in Zusammenarbeit mit dem Lehrstuhl und seinen Kooperationspartnern aufgegriffen werden.

Die zunehmenden, auch internationalen Forschungsanfragen zu hochrangigen Objekten unterstreichen die Bedeutung, die die Entwicklung der Restauratorenausbildung an der Bayerischen Staatsbibliothek genommen hat. Sie zeichnet sich durch einen signifikanten Zuwachs an Kompetenzen und an Vernetzung mit den schon länger auch wissenschaftlich arbeitenden und forschenden Restaurierungsabteilungen anderer Münchener Institutionen aus. Die Bayerische Staatsbibliothek geht daher optimistisch auf diese jüngste und vielleicht einschneidendste Veränderung ihrer Restauratorenausbildung in der Kooperation mit der TUM ein. Optimistisch vor 
allem auch deshalb, weil die in der deutschen Ausbildungslandschaft einzigartige Verbindung von Ausbildung und Restaurierungswerkstatt einer großen Institution mit profunden Beständen weiterhin der Garant für ein praxisnahes Studium mit einem großen Anteil an manueller Arbeit bleibt.

\section{Fazit: \\ Die Restauratorenausbildung am IBR im Kontext}

München bietet für die Ausbildung in allen Restaurierungssparten ideale Voraussetzungen, weil die Studierenden an den Restaurierungsabteilungen vieler bedeutender Gedächtnisinstitutionen in Lehre und Praxis ausgebildet werden und auch interdisziplinär studieren können. Die Bayerische Staatsbibliothek mit ihrem auch international vernetzten IBR als Teil dieses „Restaurierungscampus“ bildet Restauratoren für Grafik, Archiv- und Bibliotheksgut in einem im deutschsprachigen Raum einzigartigen Modell aus. Die Stärken dieses Modells liegen auf der Hand: Theorie und Praxis können die reichen und einzigartigen Bestände integrieren und auf diese Weise die Lehrinhalte an Beispielen systematisch vermitteln. Die Dozenten im IBR stehen in kontinuierlichem Bezug zur Restaurierungspraxis und Bestandserhaltung der Bayerischen Staatsbibliothek als einer der bedeutendsten Gedächtnisinstitutionen des schriftlichen Kulturerbes weltweit. Projekt- und Forschungsthemen können auf bibliotheksrelevante Fragestellungen fokussiert werden. Restauriert wird staatlicher Bestand, womit zugleich die aufwendige externe Objektakquise entfällt, die eine systematische Ausbildung nach bestimmten Schadensbildern und Restaurierungsmethoden erschwert.

Mit dem inhaltlichen Schwerpunkt „Buch und Papier“ komplettiert die TUM ihr Studienangebot um die Konservierungswissenschaft des schriftlichen Kulturerbes und damit um ein bis dahin noch fehlendes, wichtiges Modul. Die TUM hat somit als deutschlandweites Alleinstellungsmerkmal einen Studiengang für sämtliche Bereiche des Kulturerbes bis hin zur Denkmalpflege. Studierende können sich auf Gemälde, Skulpturen oder archäologische Objekte wie überhaupt auf alle Arten von Kunst- und Kulturobjekten spezialisieren und eben auch - dank der Kooperation mit der Bayerischen Staatsbibliothek seit dem Wintersemester 2009/2010 - auf das schriftliche Kulturerbe in der Vielfalt seiner Materialien und Techniken.

Für die Bayerische Staatsbibliothek konnte in der Kooperation mit der TUM die Restauratorenausbildung als ein Alleinstellungsmerkmal auch nach der universitären Studienreform im Rahmen des Bologna-Prozesses gesichert und durchgängig auf ein akademisches Niveau gehoben werden. Damit ist nicht nur ein kontinuierliches Nachwachsen an hochqualifizierten Restauratoren gewährleistet, gleichzeitig eröffnen sich vielfältige Optionen im Bereich der immer stärker nachgefragten Forschungsfelder der Kunsttechnologie und Archäometrie. Dieser Zugewinn an Mög- 
lichkeiten im IBR unterstützt zugleich die Erforschung der vielfältigen Sammlungen der Bayerischen Staatsbibliothek und des bayerischen Bibliothekswesens insgesamt. Durch Forschungsergebnisse aus Projekt- und Abschlussarbeiten ergeben sich neue Perspektiven für die einzigartigen historischen Sammlungsbestände, insbesondere im Bereich innovativer naturwissenschaftlicher Untersuchungsmethoden. Schriftliches Kulturerbe erschließt sich damit auf eine zeitgemäße Weise zur breiten Nutzung durch die Wissenschaft über nationale und fachliche Grenzen hinweg. 